Journal of Computer Science 7 (9): 1432-1438, 2011

ISSN 1549-3636

(C) 2011 Science Publications

\title{
Mobile Node Speed Detection Mechanism in Hierarchical Mobile Internet Protocol (IPv6)
}

\author{
Zulkeflee Kusin and Mohamad Shanudin Zakaria \\ Department of Computer Science, Faculty of Information Science and Technology, \\ Universiti Kebangsaan Malaysia, Bangi, Malaysia
}

\begin{abstract}
Problem statement: IETF has introduced Hierarchical Mobile IPv6 (HMIPv6) to support mobility problem in the next generation Internet Protocol (IPv6). The key concept behind this protocol is the usage of Mobility Anchor Point (MAP) located at any level router of network to support hierarchical mobility management and seamless handover. The distance MAP selection in HMIPv6 causes MAP overloaded as the network grows. Approach: To address the issue in designing MAP selection scheme, we proposed a dynamic speed detection mechanism that integrates with the load control mechanism. Results: From the experimental results we obtained that the proposed scheme gives better distribution in MAP load and reduced binding update cost. Conclusion/Recommendations: The Next Generation Networks (NGN) is expected to provide seamless handover in high speed wireless network environment. There is crucial need of very sophisticated protocols to support NGN QoS requirements.
\end{abstract}

Key words: Mobility Anchor Point (MAP), MAP selection scheme, speed detection, load control, Next Generation Networks (NGN)

\section{INTRODUCTION}

Without specific support for mobility in IPv6, packets destined to a Mobile Node (MN) would not be able to reach it while the $\mathrm{MN}$ is away from its home IP subnet. Mobile Internet IPv6 (MIPv6) allow transparent routing of IPv6 packets to MNs. In MIPv6 all packets sent to an MN must be routed first to the MN's home subnet and then forwarded to the $\mathrm{MN}$ at its current location by its Home Agent (HA).

Although MIPv6 supports mobility, it has problems on supporting seamless handover due to high delay. Every time $\mathrm{MN}$ move to new access router, it acquires new Care-of Address (CoA) and must notify Binding Update (BU) to HA and Correspondent Node $(\mathrm{CN})$ for each handover. The delay cannot be avoided when the distance growing among MN and it's HA.

Hierarchical Mobile IPv6 (HMIPv6) (Soliman et al., 2008) is an extension to Mobile IPv6 which aims to reduce the amount of signalling between the $\mathrm{MN}$ and its CNs during a handover and to improve the performance in terms of handover speed. By the usage of a new node called Mobility Anchor Point (MAP), it can support a micro-mobility management. Therefore the furthest MAP selection in HMIPv6 can be a MAP overload and increase frequent binding update problem as the network grows. It is only suitable for fast MNs that will perform frequent handoffs because the MNs reduce the changing of MAPs. Hence, without specific an efficient MAP selection scheme can affect the system performance and supporting seamless handover.

This study investigates an improvement of MAP selection scheme by MN operation in HMIPv6 (Soliman et al., 2008). The new model is proposed to reduce the $\mathrm{BU}$ delay and to achieve the network performance. Besides, we also improve the distancebased enhanced with speed detection to achieve MAP load control management in HMIPv6.

Hierarchical mobile IPv6 (HMIPv6): The design of MIPv6 does not attempt to solve all general problems related to the use of MNs or wireless networks. Specifically this protocol does not solve local or hierarchical forms of mobility management. Since MIPv6 only support global mobility, a hierarchical scheme that separates micro-mobility from macromobility is preferable. Discussed the MIPv4 and MIPv6 operation in IP mobility. The protocols need to be worked especially on the security and routing issues.

In HMIPv6 the usage of a new node, MAP can be used to improve the performance of Mobile IPv6 in terms of handover speed. An MAP is essentially a local HA situated in the foreign network as shown in Fig. 1. It can be located at any level in a hierarchical network of routers so that it can be classified as a micro-mobility.

Corresponding Author: Zulkeflee Kusin, Department of Computer Science, Faculty of Information Science and Technology, University Kebangsaan Malaysia, Bangi, Malaysia 


\section{J. Computer Sci., 7 (9): 1432-1438, 2011}

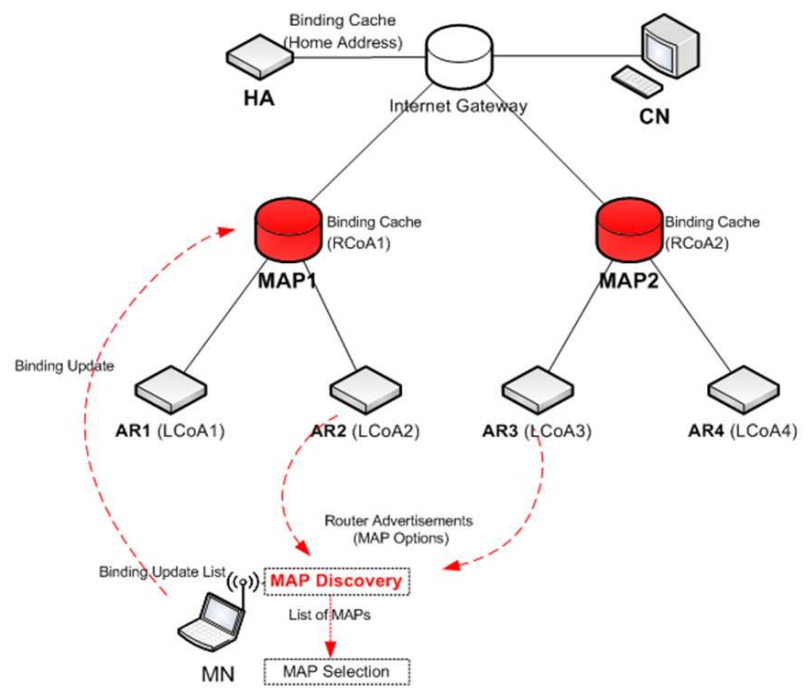

Fig. 1: HMIPv6 operations

MAP Discovery should choose to use HMIPv6 implementation if the MN is HMIPv6-aware. Besides the uses of MAP in HMIPv6, an MN will also have to configure two new types CoAs: a regional care-ofaddress (RCoA) and an on-link care-of-address (LCoA). The LCoA is a local address to the MN received from Access Router (AR). The RCoA is an address on the MAP's subnet, configured when an MN received a Router Advertisement (RA) message with the MAP Option during MAP Discovery.

The MAP performs the function of a "local" HA that binds the MN's RCoA to an LCoA. After an MN get new RCoA and LCoA addresses then it sends a Local Binding Update (LBU) to the MAP in order to establish a binding between the RCoA and LCoA.

Local Binding Update (LBU): When an MN enters a new MAP domain, it will receive RA containing information about one or more local MAPs. During RA, an $\mathrm{MN}$ will also detect whether it is still in the same MAP domain. If the MAP domain is different it needs to have two addresses from AR (LCoA and RCoA) otherwise only the LCoA will change. The MN can bind its current location (LCoA) with an address on the MAP's subnet (RCoA). The MAP will receive all packets on behalf of the $\mathrm{MN}$ it is serving and will encapsulate and forward them directly to the MN's current address.

If the MN changes its current address within a local MAP domain, it only needs to register the new LCoA with the MAP. Hence, only the RCoA needs to be registered with CNs and the HA. The RCoA does not change as long as the MN moves within a MAP domain. This makes the MN's mobility transparent to CNs it communicates with and also faster LBU compared to MIPv6.

MAP selection scheme and MAP discovery: The process of MAP Discovery continues everytime the MN received RA including a MAP option and it should start register with any new MAP through Neighbour Discovery (Narten et al., 2007). The MN needs to consider several factors to optimally select one or more MAPs, where several MAPs are available in the same domain. During this MAP selection, it will be selected that is most distant or furthest, provided that its preference value and valid lifetime did not reach a value of zero. The discovery phase will also inform the MN of the distance of the MAP from the MN and store in a MAP Option.

An MN should register with the MAP having the highest preference value. A MAP with a preference value of zero should not be used for new LBU. Also a MAP option with a valid lifetime value of zero indicates a MAP failure and when it is received, an MN must choose another MAP and create new bindings. If no other MAP is available, the MN must not attempt to use HMIPv6.

Related works: In designing MAP selection scheme, characteristics of the MAP, MN and the network topology need to be identified and discussed (Kusin and Zakaria, 2010). An MN needs to consider these elements when selecting the new MAP. Without an efficient MAP selection scheme will be seriously degrade the network performance and supporting seamless handover. Numerous researches have been carried out to deal these issues such as distance based, mobility based, adaptive based and dynamic based (Pack et al., 2004b; Hu et al., 2005; Taleb et al., 2005; Pack et al., 2006). In designing the scheme, the criteria of the MAP, MN, CN and network architecture need to be included. A technique such as load balancing or load control can be adapted to solve overload problem (Bandai and Sasase, 2003)

Pack et al (2004a) has conducted a comparative study for the above MAP selection schemes. Overall, the mobility-based and the adaptive MAP selection schemes achieve more desirable performances than the distance scheme (the furthest and the nearest schemes). Also, the adaptive MAP selection scheme performs better in terms of load balancing than the mobilitybased MAP selection scheme. 


\section{J. Computer Sci., 7 (9): 1432-1438, 2011}

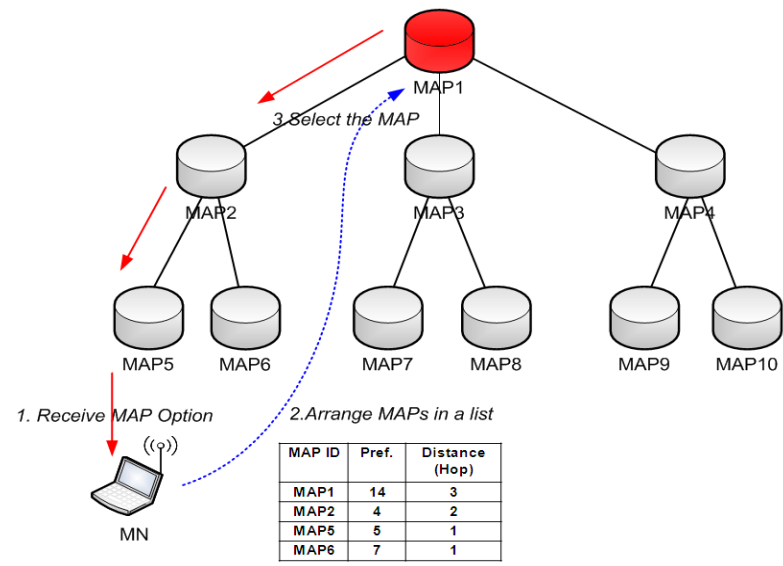

Fig. 2: Distance MAP selection scheme

Distance-based: In HMIPv6, a distance-based selection was recommended where an $\mathrm{MN}$ may choose the furthest MAP in order to avoid frequent re-registrations (Soliman et al., 2008). Figure 2 shows the process will repeat until the $\mathrm{MN}$ find the valid lifetime with a preference value of the MAP.

This algorithm is suitable for fast MNs that will perform frequent handoffs, because the fast MNs reduce the probability of changing the serving MAP and informing $\mathrm{HA}$ and $\mathrm{CNs}$ of this change. Although HMIPv6 tries to improve the binding update between local MAP and $\mathrm{CN}$, it creates the complexity of network management because of more of network entity such as MAP and additional address and LBU process.

The scheme however creates a bottleneck as the site grows larger since the corresponding MAP suffers from the overload due to the increased data traffic to be tunnelled as well as BU signalling. In this condition, registering with the furthest MAP will increase the registration delay because the hop distance between the MN and the furthest MAP is comparatively larger than that between the one and the closer MAP. The preference value set in HMIPv6 is also a static 4 bits integer and no specific procedure to set up the characteristic.

Velocity-based (speed) mechanism: In order to speed up the handover between MAPs and reduce packet loss, an MN should send a LBU to its previous MAP, specifying its new LCoA. Packets in transit that reach the previous MAP are then forwarded to the new LCoA. In a scenario where several MAPs are discovered by the $\mathrm{MN}$ in one domain, it may need sophisticated algorithms to be able to select the appropriate MAP. These algorithms would have the MN speed as an input (for distance-based selection) combined with the preference field in the MAP option (Soliman et al., 2008).

In velocity-based mechanisms (Kawano et al., 2002a; b) there are two main steps: the measurement of the MN's velocity or speed and the selection of MAP to register with.The issue is how to measure the MN's speed because it is difficult to calculate the precise value of the speed. Only when the MN's speed is estimated and then the MN can select suitable MAP by the MAP Table (MT) that records the mapping relation between the MN and related MAP. Algorithms based on the speed of an $\mathrm{MN}$, measured in handovers per unit time, were suggested in Kawano et al. (2002b). Faster MNs select more distant MAPs, as it is believed that faster movement leads to a larger moving area. Then, the estimated speed of the MT can be also obtained by dividing the distance that the MT has traversed in the previous access area by the dwell time. Joe and Lee (2009) proposed a selective MAP binding scheme that reduces the number of unnecessary local $\mathrm{BU}$ process in the MIPv6 based networks where the MN moves at high speed. Chung and Lee (2007) proposed MAP selection schemes for HMIPv6 networks: LV-MAP and DV-MAP. These two proposed schemes select an optimal MAP and the furthest MAP supporting MN's velocity, with the aim to reduce the frequency of inter-domain handovers. The MN may need sophisticated algorithms to select the appropriate MAP and its speed as an input combined with the preference field (load control value) in the MAP option during RA.

\section{MATERIALS AND METHODS}

Speed detection mechanism: The difficulty to measure the MN properties prevents the precision selecting a proper MAP and MAP is difficult to take into account of MN's properties. For the reason that MN based on its own properties is more suitable into the consideration.We suggest a model that detects the speed of the MN. The process starts with the determination of the first location to the MN's next location. The speed of the MN will be calculated by the MN with the distance value divides by the time taken during the movement between locations. The process for the speed detection can be done during the handover of the MN to the new MAP. The MNs can select the furthest and nearest MAPs by according to their speed. The fastest MNs select the most distant MAPs and vice versa. The MN also can also change the scheme dynamically whenever the speed is changed. By this the frequent $\mathrm{BU}$ and the $\mathrm{BU}$ process can be reduced. Figure 3 shows an example the distance between the previous and the new location of MN movement. 


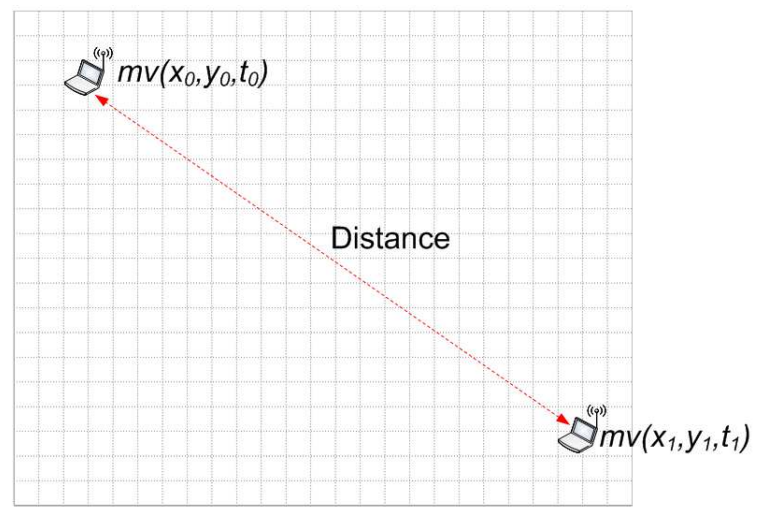

Fig. 3: MN movement

A model integrates speed detection mechanism to Distance-based MAP selection scheme which will support modularity. A few fields are introduced to MAP List (ML) as shown in Table 1. The value of field preference in MAP Option will be replaced by the load calculated from ML. In this model consist of MAP discovery process, MAP selection scheme and load control mechanism, speed detection, binding update procedure and handover process. The MAP discovery process, binding update procedure and handover process already described in the related works. In this study we only describe the proposed speed mechanism. Besides, the MAP selection mechanism also integrates with the MAP load control mechanism which improves the drawback of Wang et al., (2008).

The current load and preference value are given as:

Current load $=$ number of MAP Binding Cache

Preference $=(1-($ current load $/$

threshold value) $) * 15$

From the (2), the current load is inversely proportioned preference value. In this selection scheme the process will select the nearest MAP with highest preference where the maximum value is 15 in the MAP option.

MN movement and speed: The format of data movement is consists with value of column $(\mathrm{m})$, row (m) and time (sec). If the MN moves from current location to destination location with $\mathrm{n}$ movement then the input data will be $n$. The input will be the MN's movement from current location to destination location:

$\mathrm{mv}_{0}\left(\mathrm{x}_{0}, \mathrm{y}_{0}, \mathrm{t}_{0}\right) \rightarrow \mathrm{mv}_{\mathrm{n}}\left(\mathrm{x}_{\mathrm{n}}, \mathrm{y}_{\mathrm{n}}, \mathrm{t}_{\mathrm{n}}\right)$ where:

$\mathrm{mv}_{\mathrm{n}} \quad=$ The movement of the $\mathrm{MN}$

$\mathrm{x}_{\mathrm{n}}$ and $\mathrm{y}_{\mathrm{n}}=$ Destination coordinates of $\mathrm{x}$ and $\mathrm{y}$

$\mathrm{t}_{\mathrm{n}} \quad=$ Destination time arrived in second

So the distance and time between current location to destination location are measured by:

Distance $_{\mathrm{n}}^{2}=\left(\mathrm{x}_{\mathrm{n}}-\mathrm{x}_{\mathrm{n}-1}\right)^{2}+\left(\mathrm{y}_{\mathrm{n}}-\mathrm{y}_{\mathrm{n}-1}\right)^{2}$

Time $_{n}=t_{n}-t_{n-1}$

The total of overall distance of the MN can be measured with the sum of all movement from 0 to $n$ :

Totaldis tan ce $(n)=\sum_{n=0}^{\infty} \sqrt{\left(\left(x_{n}-x_{n-1}\right)^{2}+\left(y_{n}-y_{n-1}\right)\right)^{2}}$

where:

$\mathrm{x}_{2}$ and $\mathrm{y}_{2}=$ The coordinate of MN's new location

$\mathrm{x}_{1}$ and $\mathrm{y}_{1}=$ The coordinate of MN's previous location

$\mathrm{t}_{\mathrm{n}}$ are $\mathrm{t}_{\mathrm{n}-1}=$ Destination time and arrival time

From (4) and (5) then the speed in second(s) of $\mathrm{MN}$ can be derived:

speed $_{\mathrm{n}}=\left(\right.$ distance $_{\mathrm{n}} /$ time $\left._{\mathrm{n}}\right) \mathrm{mps}$

averagespeed $(n)=\sum_{n=0}^{\infty}\left(\frac{\text { speed }_{n}}{\text { time }_{n}}\right) / n$

The MN speed probably changes every time it registers to new MAP. The speed detection mechanism in Fig. 4 will determine the MN speed derived from the distance and time of each movement or after receiving successful Binding Acknowledgement (BA). It can dynamically change the nearest or furthest scheme depending of the current average speed.

Performance analysis: We evaluate the performance of the proposed scheme in the context of MAP selection scheme in HMIPv6. The scenario was designed by the reason of IPv6 deployment challenges especially for the implementation in the real world scenario. The network scenario is shown in Fig. 5 and the relative parameters, in which the $\mathrm{MN}$ is moving across eight ARs in a two MAP domains where each domain contains three MAPs. 
Table 1: MAP List (ML)

\begin{tabular}{l}
$\begin{array}{l}\text { MAP ID } \\
\text { LCoA Rlobal IP Address }\end{array}$ Pref. $\quad$ Pref. value Life time in sec \\
\hline Procedure Speed_Detection( ) \\
Begin \\
After receive successful BA from MAP record the new location, \\
new arrival time \\
Calculate the distance = new location- previous location(meter) \\
Calculate the time = new arrival time- previous arrival time(ms) \\
Calculate the new speed = distance $(\mathrm{m}) /$ time(s) \\
Update previous location=new location, \\
pefine the speed of MN whether slow or fast \\
End $\quad$ \\
If speed = fast then select Furthest MAP \\
elseif speed = slow then select Nearest MAP \\
End if
\end{tabular}

Fig. 4: Speed detection algorithm

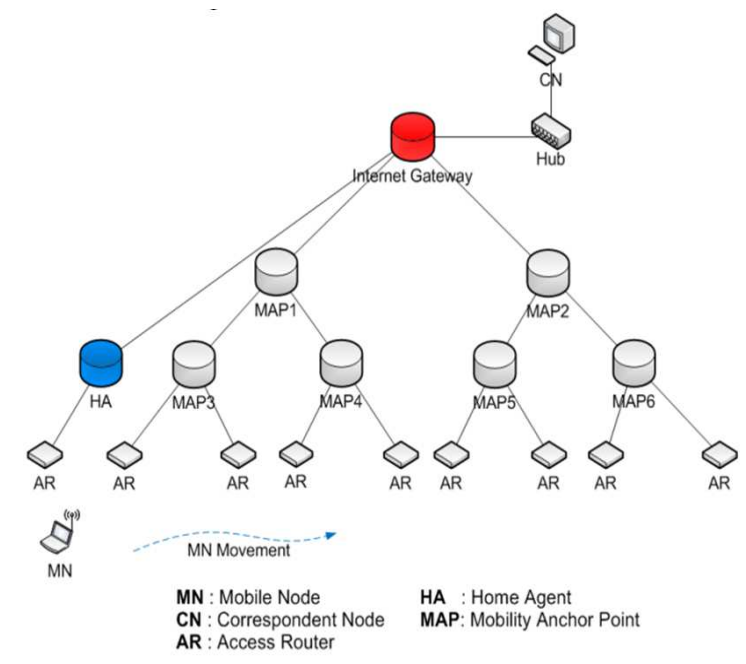

Fig. 5: Network scenario

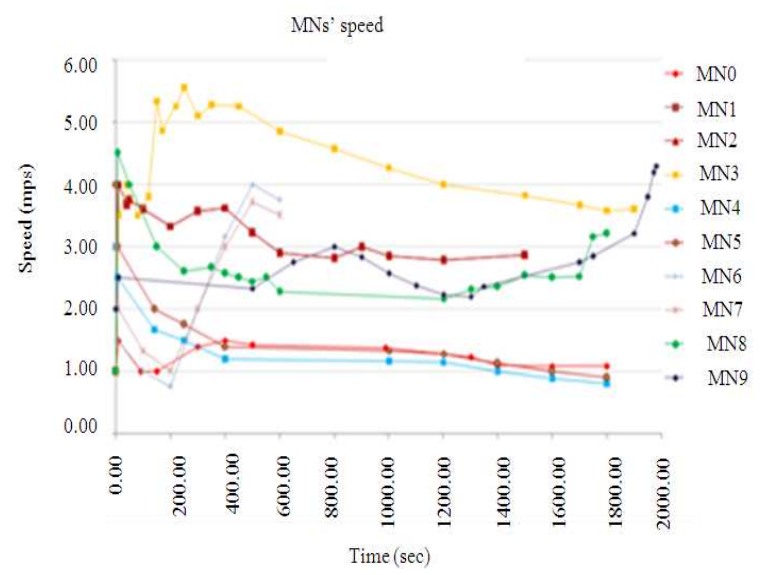

Fig. 6: MN's speed

\begin{tabular}{lll} 
Current load & Thresh-old load & Max. load \\
\hline No .of current load & No. of control load & No. of max. load \\
\hline
\end{tabular}

Experimental setup: In the simulation model, the wireless diameter is within the range $200 \mathrm{~m}$ with simulation area is $2000 \times 1250 \mathrm{~m}^{2}$. The total of ten MNs are communicated with the $\mathrm{CNs}$ through several of speed from slow to fast movement as shown in Fig. 6.

The MN starts register with the HA and undergoes intra-domain handovers as it move within same domain and inter-domain handover as it changes moving into another MAP domain. During handovers, the MN is communicating with $\mathrm{CN}$ using a TCP traffic stream and will thus establish relevant local bindings with its MAP, $\mathrm{HA}$ and the respective $\mathrm{CN}$ during the handovers. The traffics are running on ping application with 56 Bytes data and $5 \mathrm{sec}$ interval time. The wireless access network is based on the IEEE 802.11b and WLAN standard with a free space channel model. The propagation delay between the MN, the MAP, the HA and the $\mathrm{CNs}$ is assumed negligible.

For the evaluation purpose we simulate three performance metrics: load condition of each level MAP, binding update cost and ping drop by each MN. Besides, the proposed method will also be compared with the other methods: distance-based (nearest and furthest). The performance of proposed method is evaluated by the network scenario of simulation using OMNeT++ Community Site, (2011) in Fig. 5 with the relative parameters mentioned.

\section{RESULTS AND DISCUSSION}

As explained in related works, the furthest is proposed to reduce frequent handover but it is known that the highest level MAP has largest overloads. Hence, undoubtedly, the MAP load condition of each MAP can indicate the performance of MAP load control mechanism. Figure 7 shows the load distribution of each MAP by three different schemes. Although the total binding cache of proposed scheme is higher than the furthest, it supports the best distribution of MAP load.

Figure 8 illustrates the performance of binding update list between three different schemes. It is obvious to discover that the proposed scheme can reduce the total binding update list and is better than the nearest.Especially in the best case with slow MNs move within the same domain, it is still superior because the proposed scheme possibly let each MN choose the suitable MAP which efficiently reduces the binding update cost. 


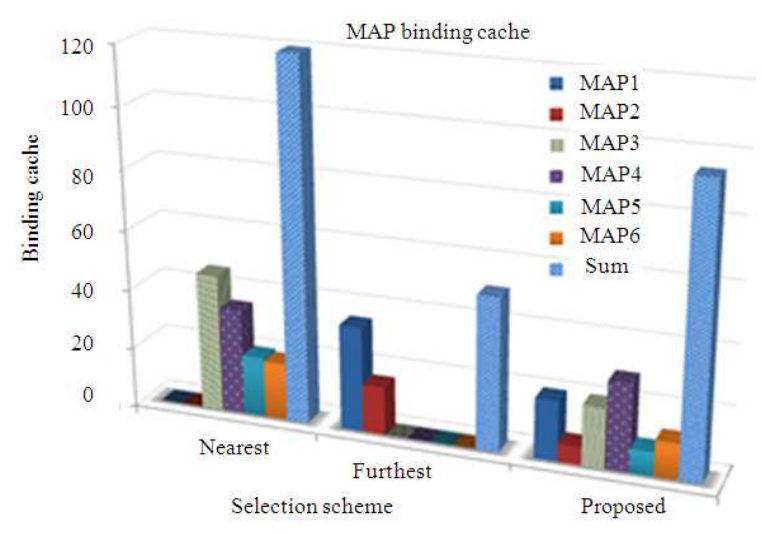

Fig. 7: Load comparison between MAPs

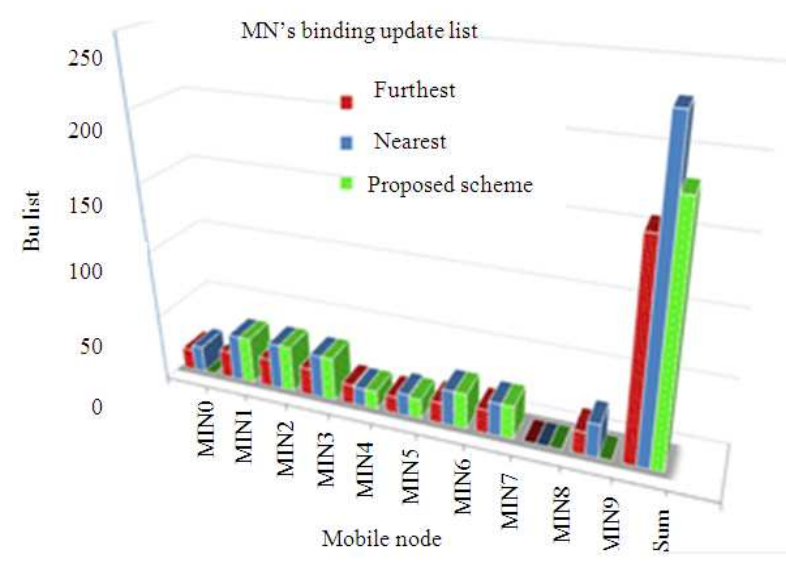

Fig. 8: Total MN's binding update list

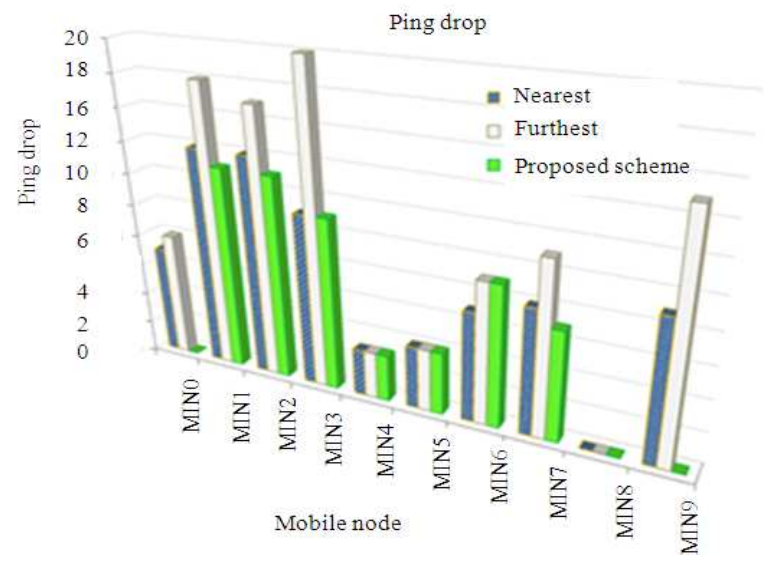

Fig. 9: Total MN's ping drop

Figure 9 illustrates the performance ping drop between three different schemes. It is obvious to discover that the proposed scheme can reduce the packet drop amongst the compared schemes. Especially in the best case with slow MNs that move within the same domain, it is still superior because the proposed scheme possibly let each MN choose the suitable MAP which efficiently reduces the packet drop

\section{CONCLUSION}

HMIPv6 protocol is one that will be support the NGN technology development for micro-mobility or Localized Mobility Management (Kempf, 2007). Previous works show that load control can reduce and relive MAPs overload. This overload is due to the increased data traffic to be tunneled as well as BU signaling and maximum number of MN connected. In this study, we discussed and proposed the speed mechanism adapted in HMIPv6 MAP selection scheme. The load control was measured based on MN and MAP properties. From the experimental results shows that our proposed scheme gives better distribution in MAP load and reduces binding update cost.

Our future work will include analysis of MAP selection scheme using load control technique with multiple speed of $\mathrm{MN}$; study on distance based algorithms using dynamic load control and form the basis for our next research.

A MAP Discovery in current HMIPv6 specification requires network administrator manually configures the MAP option information in ARs. In future, some mechanisms may be defined to allow MAPs to be discovered dynamically. The NGN is expected to provide seamless handover and also mobile entities moving in very high speed wireless network environment. There is crucial needed of very sophisticated MIPv6 and HMIPv6 protocols to support NGN QoS requirements.

\section{REFERENCES}

Bandai, M. and I. Sasase, 2003. A load balancing mobility management for multi-level hierarchical mobile IPv6 Networks. Proc. IEEE PIMRC, 1: 460-464. DOI: 10.1109/PIMRC.2003.1264314

Chung, W. and S. Lee, 2007. Improving performance of HMIPv6 networks with adaptive MAP selection scheme. IEICE Trans. Communi., 90: 769-776.

Hu, X., J. Song and M. Song, 2005. An adaptive mobility anchor point selection algorithm for hierarchical mobile IPv6. Proc, IEEE ISCIT, 2:1148-1151. DOI: 10.1109/ISCIT.2005.1567072

Joe, I. and W. Lee, 2009. A selective MAP binding scheme based on the mobile speed. Proceeding of the 5th International Joint Conference on INC, IMS and IDC, Aug. 25-27, IEEE Xplore Press, Seoul, pp: 527-530. DOI: 10.1109/NCM.2009.252 
Kawano, K., K. Kinoshita and K. Murakami, 2002a. A mobility-based terminal management in IPv6 networks. IEICE Trans. Communi, 85: 2090-2099.

Kawano, K., K. Kinoshita and K. Murakami, 2002b. A multilevel hierarchical distributed ip mobility management scheme for wide area networks. Proceeding of IEEE 11th International Conference Computer Communication and Networks, Oct. 1416, IEEE Xplore Press, USA, pp: 480-484, DOI: 10.1109/ICCCN.2002. 1043109

Kempf, J., 2007. Problem Statement for Network-Based Localized Mobility Management (NETLMM). Network Working Group

Kusin, Z. and M.S. Zakaria, 2010. An extension to mobile IPv6 micro-mobility management. Proceeding of the International Symposium in Information Technology (ITSim), June, 15-17, IEEE Xplore Press, Kuala Lumpur, 2: 1057-1060. DOI: 10.1109/ITSIM.2010.5561587

Narten, T., E. Nordmark and W. Simpson, 2007. Neighbor Discovery for IP Version 6 (IPv6). Standards Track.

Pack, S., M. Nam, T. Kwon and Y. Choi, 2006. An adaptive mobility anchor point selection scheme in hierarchical mobile IPv6 networks. Comput. Communi., 29: 3066-3078.
Pack, S., T. Kwon and Y. Choi, 2004. A comparative study of mobility anchor point selection schemes in hierarchical mobile IPv6 Networks. Proceedings of the second international workshop on Mobility management and wireless access protocols, ACM New York, USA, pp: 130-131. DOI: 10.1145/1023783.1023811

Pack, S., T. Kwon and Y. Choi, 2004. A mobility-based load control scheme at mobility anchor point in hierarchical mobile IPv6 networks. Proceeding of the Global Telecommunications Conference, 29 Nov.-3 Dec, IEEE Xplore Press, USA, 6: 34313435. DOI: $10.1109 /$ GLOCOM.2004.1379004

Soliman, H., C. Castelluccia, K. Malki and L. Bellier, 2008. Hierarchical Mobile IPv6 Mobility Management (HMIPv6). Standards Track.

Taleb, T., T. Suzuki N. Kato and Y. Nemoto, 2005. A dynamic and efficient MAP selection scheme for mobile IPv6 networks. Proceeding of IEEE Globecom Telecomunication Conference, Dec. 2-2, IEEE Xplore Press, St. Louis, MO, 5: 2891-2895, DOI: 10.1109/ GLOCOM.2005. 1578287

Wang, Y.H., K.F. Huang, C.S. Kuo and W.J. Huang, 2008. Dynamic MAP selection mechanism for HMIPv6. Proc. Int. Conf., pp: 691-696. DOI: 10.1109/AINA.2008.17 